\title{
Estimation of elevation-dependent satellite antenna phase center variations of GPS satellites
}

\author{
R. Schmid, M. Rothacher \\ Institut für Astronomische und Physikalische Geodäsie, Technische Universität München, 80290 München, Germany \\ e-mail: schmid@bv.tum.de; Tel.: +49-89-289-23193; Fax:+49-89-289-23178
}

Received: 26 September 2002 / Accepted: 19 May 2003

\begin{abstract}
A method for the estimation of the phase center variations of GPS satellite antennas using global GPS data is presented. First estimations have shown an encouraging repeatability from day to day and between satellites of the same block. Thus, two different satellite antenna patterns for Block II/IIA and for Block IIR with a range of about $4 \mathrm{~cm}$ and an accuracy of less than $1 \mathrm{~mm}$ could be found. The present approach allows the creation of a consistent set of receiver and satellite antenna patterns and phase center offsets. Thereby, it is possible to switch from relative to absolute phase center variations without a scale problem in global networks. This changeover has an influence on troposphere parameters, reduces systematic effects due to uncorrect antenna modeling and should diminish the elevation dependence of GPS results.
\end{abstract}

Keywords: Global positioning system - Satellite antenna - Absolute phase center variations - Global scale

\section{Introduction}

It is well known nowadays that phase center variations of the receiver antenna have to be taken into account in combination with the corresponding phase center offsets in order to obtain high-precision GPS results. For short baselines relative phase center variations (patterns relative to the reference antenna Dorne Margolin T) currently applied by the International GPS Service (IGS) are sufficient. In global networks however, absolute phase center variations have to be taken into account due to the fact that the GPS satellites are normally seen at different elevations from the ends of a baseline (Mader 1999). Apart from that, it is not

Correspondence to: R. Schmid satisfactory that for the reference antenna (Dorne Margolin T) phase center variations are assumed to be zero. For this reason several methods have been developed in order to determine absolute phase center variations: anechoic chamber measurements and absolute calibrations in the field. Although Rothacher (2001) and others have shown good agreement between values derived from these independent approaches, the absolute variations are still not in use. The IGS was reluctant to introduce them because they cause an unreasonably large terrestrial scale change in global GPS solutions of up to $15 \mathrm{ppb}$ compared to results from Very Long Baseline Interferometry (VLBI) and Satellite Laser Ranging (SLR) (see e.g. Rothacher et al. 1995; Menge and Seeber 1999; Springer 2000). This scale change points to an unmodeled systematic effect that does not show up in the results using the relative phase center variations of the IGS.

This systematic effect is most probably related to the positions of the satellite antenna phase centers. Whereas big efforts were made to examine the behavior of the receiver antenna phase centers (development of measuring robots, estimation of azimuth-dependent variations), very little is known about the satellite antenna phase centers. As variations with the emitting direction are even obvious in the satellite case because each antenna consists of 12 helical elements arranged in two concentric circles (Aparicio et al. 1995; cf. Fig. 1), this is all the more astonishing. So far only one offset value per satellite block (Block I, Block II/IIA, Block IIR) is known and several estimations and comparisons (e.g. by Rothacher 1994; Brockmann 1997; Zhu et al. 2003) have shown that these values are not accurately known. In addition, it is not clear whether the offset values indicate the phase center of the L1 or L2 frequency or perhaps the phase center of the ionosphere-free linear combination (LC) of L1 and L2. In order to solve the latter problem, Mader and Czopek (2002) conducted an experiment with a real Block IIA satellite antenna. They turned it upside down, used it as a receiver antenna and estimated vertical offsets for L1, L2, and LC. Furthermore, a joint working group of the IGS, the IVS 


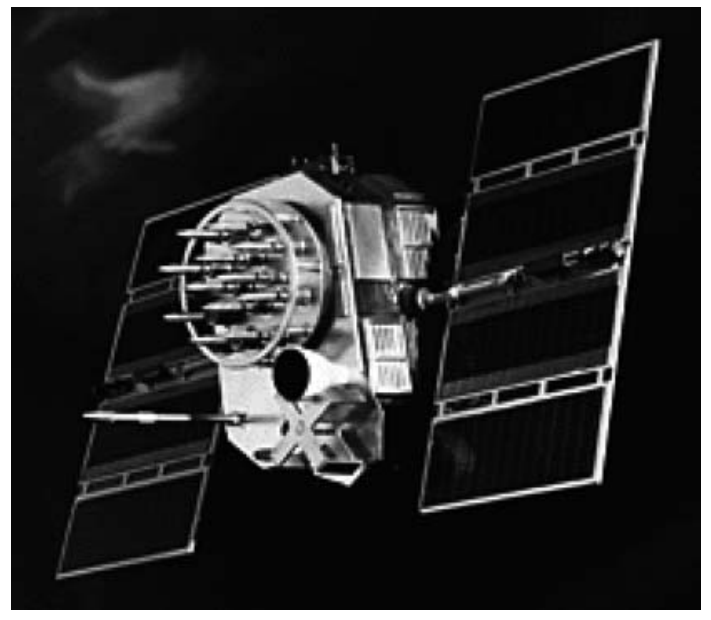

Fig. 1. Block II satellite (Courtesy of The Aerospace Corporation, El Segundo, CA)

(International VLBI Service) and the ILRS (International Laser Ranging Service) has studied whether the use of VLBI could help to obtain information about the satellite antenna phase centers. Unfortunately, this working group arrived at the conclusion that VLBI measurements in all probability will not be accurate enough.

The existence of elevation-dependent GPS satellite antenna phase center variations has been supposed by Springer (2000), Rothacher (2001), and others, but never verified. One of the major problems in this context is the high correlation between several parameters, namely clock biases, tropospheric delays, as well as phase center offsets and variations of both the receiver and the satellite antennas. The authors implemented satellite antenna phase center variation parameters depending on the elevation (satellite seen from the station), respectively the nadir angle (station seen from the satellite), into the Bernese GPS Software (Hugentobler et al. 2001), allowing grouping of satellites. Thereby it was possible to estimate phase center patterns using existent GPS data from IGS stations all over the world, i.e. without any additional measuring expenditure.

The present paper is organized as follows. After a short summary of the underlying basic relations in Sect. 2 we give some information about the data used for the LC pattern estimation in Sect. 3. In Sect. 4 we show that the results are quite encouraging with regard to the repeatability from day to day and between satellites of the same block, and how other simultaneously estimated parameters (troposphere, orbit and Earth rotation parameters) are affected by the estimation of such satellite antenna patterns.

\section{Basic relations}

In theory, the equiphase wavefront from a transmitting antenna should be perfectly spherical. In practice, however, there are deviations from perfect sphericity. These deviations, expressed as a function of angular position about the antenna, are called the "phase pattern'. The same name is used for the process of signal reception, although in that case the pattern describes the radial deviations from a perfectly punctual receiver antenna phase center.

An easy one-to-one relationship exists between the elevation-dependent fractions of the two patterns (satellite and receiver antenna) (Rothacher 2001). The nadir angle $z^{\prime}$ at the satellite is related to the zenith angle $z$ for the receiver at the ground by

$\sin \left(z^{\prime}\right)=\frac{R}{r} \sin (z)$

where $R$ is the Earth's radius and $r$ the geocentric distance of the satellite (see Fig. 2). Whereas the zenith angle $z$ at the receiver ranges from 0 to $90^{\circ}$, the corresponding nadir angle $z^{\prime}$ as seen from the satellite only varies between 0 and approximately $14.28^{\circ}$. This maximum value

$z_{\max }^{\prime}=\arcsin \left(\frac{R_{\max }}{r_{\min }} \cdot \sin \left(z_{\max }\right)\right)$

can only be obtained for stations at high altitude $\left(R_{\max } \approx 6387 \mathrm{~km}\right)$ and for satellites close to the perigee in an orbit with comparatively high eccentricity $\left(e_{\max } \approx 0.025\right)$. If an elevation cut-off angle of $10^{\circ}$ is applied by the receiver, the nadir angle is always below $14.06^{\circ}$. With the aid of the nadir angle $z^{\prime}$ an elevationdependent phase center pattern $\phi(z)$ of the receiver antenna may be interpreted as a phase center pattern $\phi^{\prime}\left(z^{\prime}\right)$ of the satellite antenna, and vice versa, with

$\phi^{\prime}\left(z^{\prime}\right)=\phi(z)$

A change $\Delta \phi(z)$ of the receiver antenna pattern affects the satellite antenna pattern with reversed algebraic sign

$\Delta \phi^{\prime}\left(z^{\prime}\right)=-\Delta \phi(z)$

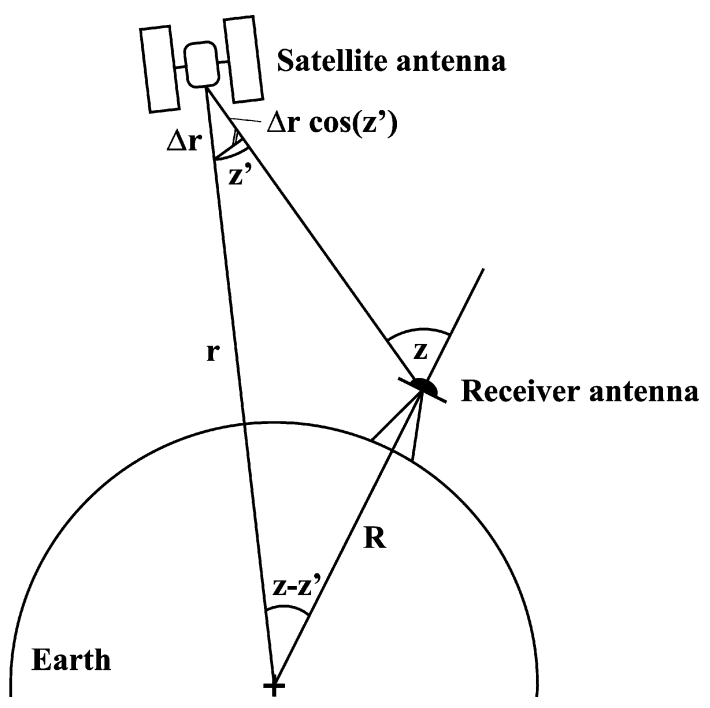

Fig. 2. Relationship between satellite and receiver antenna phase center variations, respectively between phase center offset and pattern 


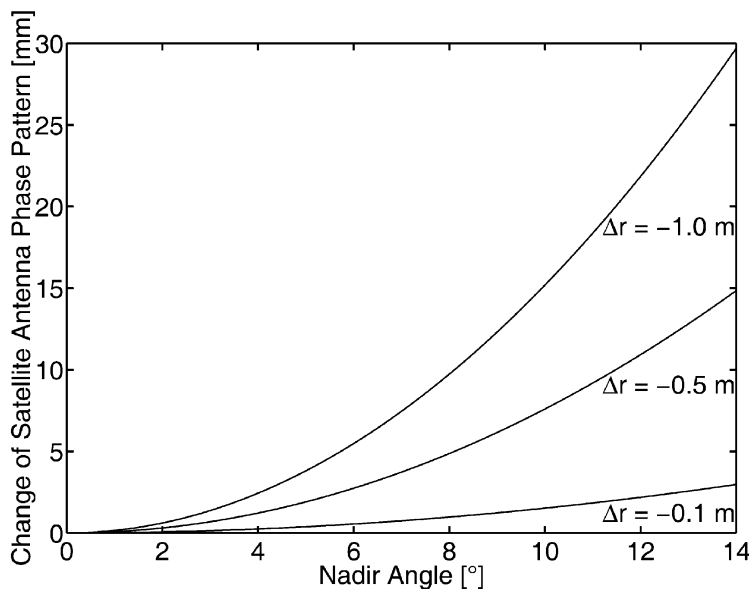

Fig. 3. Change $\Delta r$ of the phase center offset interpreted as a change $\Delta \phi^{\prime}\left(z^{\prime}\right)$ of the phase pattern

Another correlation exists between phase center offsets and variations. In the satellite case a change $\Delta r$ of the antenna phase center offset in the nadir direction can be interpreted as a cosine-dependent change of the phase pattern that is given by (see Fig. 2)

$\Delta \phi^{\prime}\left(z^{\prime}\right)=-\Delta r \cdot\left(1-\cos \left(z^{\prime}\right)\right)$

Due to the small range of the nadir angle, a change of the offset value has only a small impact on different emitting directions (see Fig. 3). The main part of the offset change is absorbed by the satellite clock estimate. Of course, such a dependence can also be found for the receiver.

Thus, we have four fully correlated parameter groups (satellite and receiver antenna phase center offsets and variations) that cannot be estimated simultaneously from GPS data, i.e. three of the four have to be fixed. Furthermore, not only receiver antenna phase center offset and variation values have to be used consistently: introducing satellite antenna phase center offset and variation parameters requires four consistent parameter sets.

Besides the elevation-dependent phase center variations, azimuth-dependent ones also have to be expected, because each satellite antenna consists of 12 single elements. In order to study these, the attitude of the satellite would have to be taken into account, namely the rotation about the $z$-axis that points towards the Earth. Although this information is also necessary for the offset correction, the authors restricted their investigation to elevation-dependent variations for the time being.

Elevation-dependent phase center variations can be modeled as a piecewise linear function (polygon approach). The use of spherical harmonic functions (see e.g. Hugentobler et al. 2001) would only make sense if azimuth-dependent variations were also involved. The authors modified the Bernese GPS Software in order to be able to estimate the parameters of the polygon approach, whereupon one parameter per polygon point is set up. The number of polygon points, i.e. the resolution of the pattern, can be chosen arbitrarily. Apart from that, the software allows grouping of satellites, whereby it is possible to estimate one single pattern for a group of satellites carrying the same antenna. Due to a correlation with the satellite clock error that does not depend on the emitting direction, an arbitrary constant of the pattern cannot be estimated. In order to prevent the normal equation system from becoming singular the sum of all pattern values is constrained to be zero

$\sum_{z^{\prime}=0^{\circ}}^{14^{\circ}} \phi^{\prime}\left(z^{\prime}\right)=0$

A second possibility would have been to constrain the value in the nadir direction to be zero $\left[\phi^{\prime}\left(z^{\prime}=0^{\circ}\right)=0\right]$.

\section{Input data}

The authors estimated one-day solutions using doubledifference phase data from more than 100 global IGS stations for the days 195-200 (14-19 July) of the year 2002. Further input data sets were a coordinate file, daily orbit files (one-day solutions from CODE, the Center for Orbit Determination in Europe) as a priori information and, finally, receiver and satellite antenna information files (cf. Sect. 2).

For the satellite antennas the official phase center offset values of the IGS (see Table 1) were adopted, for the receiver antennas two different calibration sets were considered, in each case phase center offsets and variations. In order to be able to estimate realistic phase patterns for the satellite antennas absolute receiver antenna phase patterns had to be introduced, whereas for the investigation of the effect of that estimation on other parameters, relative values were necessary (cf. Sect. 4.3). The absolute patterns were provided by Prof. G. Seeber's group (University of Hannover), which has established a new method for their determination together with the company Geo ++ (Menge et al. 1998). They use the GPS data collected by two receivers on a short well-known baseline. In order to obtain absolute patterns the antenna to be calibrated is constantly rotated and tilted by a robot. The relative patterns used by the authors were those provided by the IGS (ftp://igscb. jpl.nasa.gov/igscb/station/general/igs_01.pcv).

\section{Results}

\subsection{Distribution of observations}

For a better understanding of the results, first the distribution of the observations per day with respect to the different satellite blocks and with respect to the nadir

Table 1. IGS satellite antenna phase center offset values

\begin{tabular}{llll}
\hline Satellite block & $\Delta x(\mathrm{~m})$ & $\Delta y(\mathrm{~m})$ & $\Delta z(\mathrm{~m})$ \\
\hline Block II/IIA & 0.2790 & 0.0000 & 1.0230 \\
Block IIR & 0.0000 & 0.0000 & 0.0000 \\
\hline
\end{tabular}


angle was analysed. For 19 July, for instance, data of 138 IGS stations were used for the estimation process. Using an elevation cut-off angle of $3^{\circ}$ and a sampling rate of 10 minutes the data yielded more than 300000 observations altogether. The distribution concerning the satellite blocks (see Table 2) is thereby roughly proportional to the number of satellites per block. The distribution with respect to the nadir angle (see Fig. 4) is, up to approximately $10^{\circ}$, in good agreement with the proportion of the area of the rings generated by two consecutive nadir angles on the surface of the Earth. (The larger the area of the ring, the more stations that are likely to be found within it.) Above a nadir angle of $10^{\circ}$ there are less observations than expected, which could be due to the non-uniform distribution of permanent GPS stations on the Earth's surface (most stations in Europe and Northern America, only few stations in polar regions). It is notable that the number of observations decreases above $13.5^{\circ}$ although an elevation cut-off angle of $3^{\circ}$ (corresponding to nadir angles of 13.48 to $14.26^{\circ}$ ) is applied. Not all IGS stations had recorded observations up to an elevation angle of $3^{\circ}$, and the maximum nadir value can only be obtained under special circumstances (high eccentricity, satellite close to the perigee) as mentioned in Sect. 2. All observations with a nadir angle beyond $14^{\circ}$ were ignored (only $0.02 \%$ of all observations on $19 \mathrm{July}$ ).

\subsection{Estimation of satellite antenna phase center patterns}

The daily patterns were estimated together with site coordinates, site-specific troposphere parameters, orbit

Table 2. Distribution of observations with respect to the satellite blocks (19 July 2002)

\begin{tabular}{lrl}
\hline Satellite block & Number & Percentage \\
\hline Block II (4 satellites) & 43964 & 14.1 \\
Block IIA (18 satellites) & 200904 & 64.3 \\
Block IIR (6 satellites) & 67601 & 21.6 \\
\hline
\end{tabular}

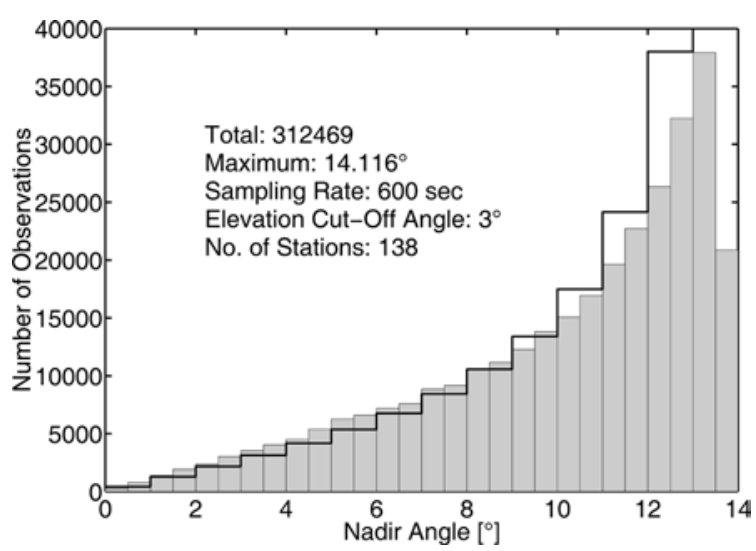

Fig. 4. Distribution of observations with respect to the satellite nadir angle (19 July 2002). The solid black line denotes the proportion of the area of the rings generated by two consecutive nadir angles on the surface of the Earth parameters and Earth rotation parameters. About $75 \%$ of the ambiguities were fixed. To prevent the normal equation system from becoming singular the stations belonging to the coordinate set IGS01P37_RS54.SNX were constrained to $1 \mathrm{~mm}$ in all components. Thus, the scale of the global network was fixed to the ITRF2000 $($ ITRF $=$ International Terrestrial Reference Frame) scale (respectively to the coordinate set IGS01P37_RS54.SNX available at ftp://igscb.jpl.nasa. gov/igscb/station/coord/). Orbit and Earth rotation parameters had to be estimated, because the a priori values assumed the satellite antenna phase center variations to be zero. For each satellite the six orbital elements, five radiation pressure coefficients [corresponding to the X3 solution in Springer et al. (1999)] and one threedimensional (3-D) stochastic pulse at 12 UT were set up (14 parameters per satellite altogether). Earth rotation was modeled by a set of six parameters: offset and drift for two pole coordinates and for the difference between UT1 and UTC. In order to remove the influence of the tropospheric refraction the Dry Niell mapping function was used to map the dry zenith delay derived from a standard atmosphere using the formula of Saastamoinen (1973) and every 3 hours (eight parameters per day) one zenith delay correction per station was estimated using the Wet Niell mapping function (see also Hugentobler et al. 2001, p. 188). The ionospheric refraction was eliminated by forming the ionosphere-free linear combination LC. Thus, it was not possible to estimate separate L1 and L2 satellite phase patterns. Absolute receiver antenna phase patterns were introduced and, as already mentioned in Sect. 4.1, an elevation cut-off angle of $3^{\circ}$ and a sampling rate of 10 minutes were applied.

According to Aparicio et al. (1995) there should be no difference between the antennas carried by Block II satellites and by Block IIA satellites. This fact could be proved by an estimation of separate patterns for the two satellite blocks which generally agreed on the millimeter level. Consequently the satellites of the two blocks were combined and only one single phase pattern for all Block II/IIA satellites was estimated in all following investigations.

As mentioned in Sect. 2, the number of polygon points over the range of $14^{\circ}$ is an input option in the estimation program. In order to study the influence of the resolution of the pattern on the results, patterns with a resolution of 2,1 , and $0.5^{\circ}$ were estimated. The higher the resolution, the more outliers for low and high nadir angles occurred due to the small number of observations (cf. Fig. 4). In the remaining range of values the curves were nearly congruent. As the $2^{\circ}$ patterns were somewhat too smooth, a resolution of $1^{\circ}$ proved reasonable.

In order to check the repeatability between different satellites carrying the same antenna, daily patterns of single satellites of the same satellite block were compared to a pattern estimated using the observations of all satellites of the block. Figure 5 shows the patterns for all individual satellites of Block IIR and the block-specific pattern of day 200. The small differences of up to only $3 \mathrm{~mm}$ legitimate the use of block-specific patterns only. 


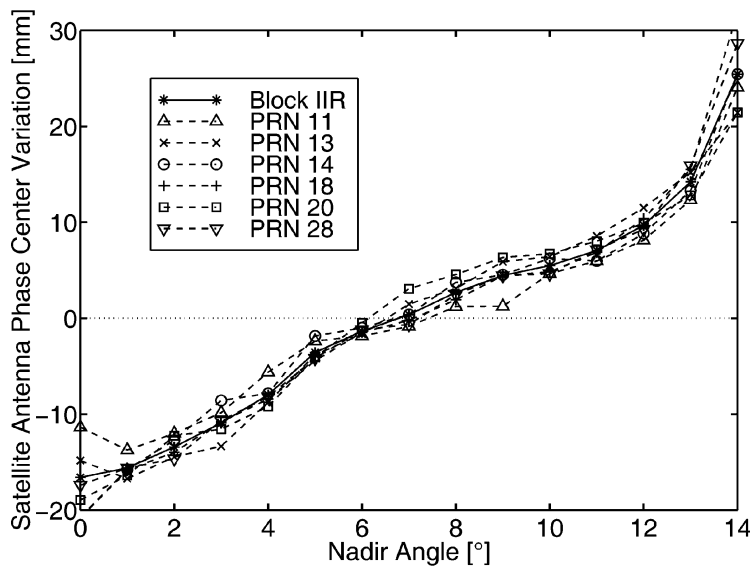

Fig. 5. Satellite antenna phase pattern LC for individual satellites of Block IIR (19 July 2002)

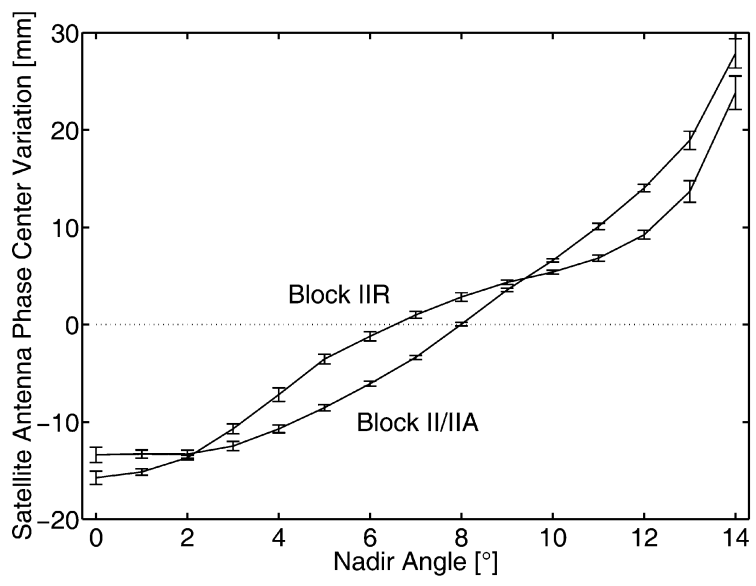

Fig. 6. Mean satellite antenna phase pattern LC with standard deviation (average over 6 days)

Estimating block-specific patterns for six consecutive days gives an impression of the repeatability from day to day: daily solutions only differ by $1-2 \mathrm{~mm}$. Figure 6 shows the result of an averaging of the six daily solutions for Block II/IIA and for Block IIR. Up to a nadir angle of $13^{\circ}$ the standard deviation is always below $1 \mathrm{~mm}$, whereas for a nadir angle of $14^{\circ}$ a worse value is obtained. The latter problem is due to the decrease in observations and the error budget of low-elevation observations (multipath, ionospheric and tropospheric refraction). The pattern for Block II/IIA shows a strong cosine dependence that denotes the use of a non-optimal value for the phase center offset. Since the pattern covers a range of about $4 \mathrm{~cm}$, the phase center offset in the

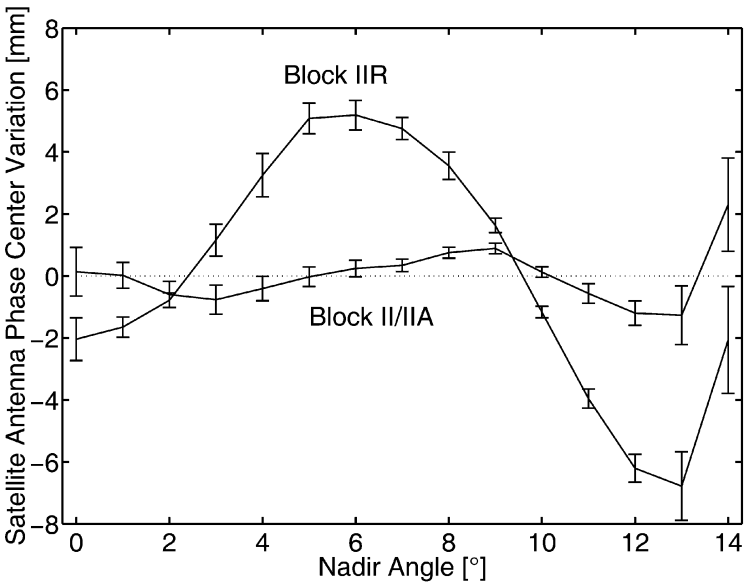

Fig. 7. Mean satellite antenna phase pattern LC after elimination of the cosine-dependent fraction

Table 3. Satellite antenna phase center LC offset values corresponding to Table 4

\begin{tabular}{llll}
\hline Satellite block & $\Delta x(\mathrm{~m})$ & $\Delta y(\mathrm{~m})$ & $\Delta z(\mathrm{~m})$ \\
\hline Block II/IIA & 0.2790 & 0.0000 & 2.3384 \\
Block IIR & 0.0000 & 0.0000 & 1.3326 \\
\hline
\end{tabular}

$z$-direction (pointing towards the Earth) would have to be changed by $\Delta r_{\mathrm{II} / \mathrm{IIA}}=+131.5 \mathrm{~cm}$, in order to eliminate the cosine-dependent fraction of the phase center variation (cf. Fig. 3). For comparison, Mader and Czopek (2002) estimated a correction of about $+63.3 \mathrm{~cm}$ for the LC $z$ offset, but they used the data up to a zenith angle of $30^{\circ}$. An elimination of the cosine-dependent fraction is also possible for the Block IIR pattern $\left(\Delta r_{\text {IIR }}=+133.3 \mathrm{~cm}\right)$, but it is important to mention that the significantly different behavior of the two satellite blocks remains, as can be seen in Fig. 7. The fact that the two numerical values for $\Delta r$ are in good agreement indicates, however, that the satellite antenna offsets for Block II/IIA and Block IIR used by the IGS so far are quite consistent. Tables 3 and 4 show the absolute values for the phase center offsets and variations of the GPS satellite antennas that will go into the new IGS antenna file.

Using relative phase center variations for the receiver antennas, at least something like a weighted mean (proportional to the number of satellites per block) of the two curves in Figs. 6 and 7 was unconsciously taken into account via Eq. (4). It was not possible, however, to compensate for the difference between the two blocks. The fact that this difference can be considered now is, apart from the more correct patterns, the reason for
Table 4. Mean satellite antenna phase center variations (PCV) LC after elimination of the cosine-dependent fraction

\begin{tabular}{llllllllllllllll}
\hline $\begin{array}{l}\text { Nadir } \\
\text { angle }\left({ }^{\circ}\right)\end{array}$ & 0 & 1 & 2 & 3 & 4 & 5 & 6 & 7 & 8 & 9 & 10 & 11 & 12 & 13 & 14 \\
\hline $\begin{array}{c}\text { Block II/IIA } \\
\text { PCV (mm) }\end{array}$ & 0.1 & 0.0 & -0.6 & -0.8 & -0.4 & 0.0 & 0.2 & 0.3 & 0.8 & 0.9 & 0.1 & -0.6 & -1.2 & -1.3 & 2.3 \\
$\begin{array}{c}\text { Block IIR } \\
\text { PCV (mm) }\end{array}$ & -2.0 & -1.6 & -0.8 & 1.2 & 3.3 & 5.1 & 5.2 & 4.8 & 3.6 & 1.6 & -1.2 & -4.0 & -6.2 & -6.8 & -2.1 \\
\end{tabular}




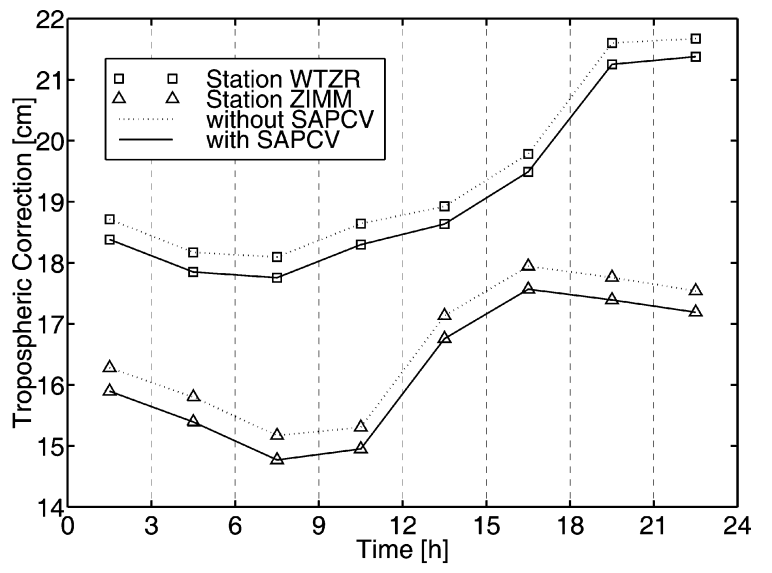

Fig. 8. Effect of the estimation of satellite antenna phase center variations (SAPCV) on the estimated tropospheric zenith delay corrections with respect to the a priori dry delay (two stations, 14 July 2002)

some changes in global GPS parameters (Earth rotation, orbits, troposphere, cf. Sect. 4.3) after switching to absolute phase center variations for both the receivers and the satellites.

Finally, it is worth mentioning that the satellite antenna phase patterns depend very much on the adopted global scale. The difference between the first $\left(z^{\prime}=0^{\circ}\right)$ and the last $\left(z^{\prime}=14^{\circ}\right)$ pattern value changes by about $4.8 \mathrm{~mm} / \mathrm{ppb}$ when scaling the set of global IGS station coordinates. This means that GPS will not be able to determine the global scale as long as it is not possible to accurately calibrate the satellite antennas by other means than by global GPS measurements. One possibility would be a calibration in an anechoic chamber before the launch. Due to the scale dependence the authors decided to fix the station coordinates to the ITRF2000 scale stemming from VLBI and SLR. The fact that the use of relative receiver antenna patterns provided a scale close to the ITRF scale was a fortunate coincidence which had to do with the choice of the satellite antenna offset values and of the antenna Dorne Margolin $\mathrm{T}$ as the reference antenna.

\subsection{Impact on global GPS parameters}

An interesting question is whether and how global GPS parameters such as Earth rotation, orbit and troposphere parameters are affected by the introduction of satellite antenna phase center variations. An ideal procedure would have been to compare one set of parameters estimated under actual conditions (relative phase center variations for the receiver antenna, no correction for the satellite antenna) with a second set estimated as it will be done in the near future, i.e. using absolute patterns for both the receiver and the satellite antenna. The problem is that errors in the relative and/ or in the absolute calibration of individual receiver antennas would affect the results. If we are only interested in the effect of the satellite antenna phase center variations another opportunity arises: estimating one set of parameters together with these variations and another one without them while using relative receiver antenna patterns in both cases. The approach of estimating absolute phase center variations for the satellite antennas while using relative receiver antenna patterns is allowed because of Eq. (4). The only unaesthetic thing is that not all corrections are added at the correct place, because the relative patterns also contain variations of the satellite antenna phase centers (cf. Sect. 4.2).

For the examination of the tropospheric delay, eight site-specific troposphere parameters (correction values with respect to the a priori dry delay) per day were estimated. Highly correlated with the antenna parameters (cf. Sect. 1), the tropospheric zenith delay shows a systematic decrease of about $4-5 \mathrm{~mm}$ for all sites, as can be seen in Fig. 8 where the results for two stations are given.

In the analysis of the Earth rotation parameter series no systematic changes could be found. The same is true for the orbit parameters. The GPS satellite orbits estimated together with satellite antenna phase center variations agree with those estimated without them on the overall orbit accuracy level. (Systematic changes of the Block II/IIA orbits that had been found in the course of the authors' first investigations were due to some orbit parameter constraints that were initially too tight.)

\section{Conclusion}

It has been shown that it is possible to estimate the antenna phase center variations (and indirectly also the offsets) of GPS satellites from global GPS measurements. Thus, a consistent set of receiver and satellite antenna patterns and offsets is available, adopting the ITRF2000 scale. The values gained from the estimation allow switching from relative to absolute receiver antenna phase center variations, as the IGS has already decided to do.

At the time of changeover, jumps in the time series of several global GPS parameters (e.g. troposphere and site coordinates) have to be expected (cf. Sect. 4.3). However, the ambiguity resolution should benefit from the more correct modeling of the phase centers of both the receivers and the satellites. The radial bias of about $5 \mathrm{~cm}$ between GPS and SLR that is observed for two Block IIA satellites (PRN5 and 6) is apparently not due to the satellite antenna patterns, as no systematic change of the orbits could be demonstrated.

Due to the fact that there are two different satellite antenna patterns (differences of up to $6 \mathrm{~mm}$ between Block II/IIA and Block IIR), it would never have been possible to compensate for that effect by confining ourselves to receiver antenna patterns only. The latter approach caused systematic effects, particularly showing up in elevation-dependent coordinate and troposphere results (results depending on the elevation cut-off angle). This problem should disappear at least partially. 
It is worth mentioning once again that GPS is not able to determine the global scale at the moment. The strong dependence of the satellite antenna patterns on the global scale and vice versa requires an independent highprecision calibration method for the satellite antennas, which is a difficult task due to their dimensions.

The processing of GPS data collected by Low Earth Orbiters (LEOs) such as CHAMP or TOPEX will require satellite antenna phase pattern values for nadir angles higher than $14^{\circ}$ that are not yet available. Their data will permit estimation of the patterns with higher accuracy for high nadir angles.

Acknowledgments. The authors thank Prof. G. Seeber (University of Hannover) and Dr. G. Wübbena $(\mathrm{Geo}++\mathrm{GmbH})$ and their groups for their kindness in making available the absolute field calibration results derived from robot measurements.

\section{References}

Aparicio M, Brodie P, Doyle L, Rajan J, Torrione P (1995) GPS satellite and payload. In: Parkinson BW, Spilker JJ (eds) Global positioning system: theory and applications, vol. I. American Institute of Aeronautics and Astronautics, Inc., Washington, pp 209-244

Brockmann E (1997) Combination of solutions for geodetic and geodynamic applications of the global positioning system (GPS). Geodätisch-geophysikalische Arbeiten in der Schweiz, Vol 55, Schweizerische Geodätische Kommission, Zurich

Hugentobler U, Schaer S, Fridez P (2001) Bernese GPS Software, version 4.2. Astronomical Institute, University of Berne
Mader GL (1999) GPS antenna calibration at the National Geodetic Survey. GPS Solut 3(1): 50-58

Mader GL, Czopek FM (2002) The Block IIA satellite - calibrating antenna phase centers. GPS World 13(5):40-46

Menge F, Seeber G (1999) Untersuchungen und Beiträge zur Problematik der Phasenzentrumsvariationen von GPS-Antennen. Deutsche Geodätische Kommission, B310, München, pp 181-194

Menge F, Seeber G, Völksen C, Wübbena G, Schmitz M (1998) Results of absolute field calibration of GPS antenna PCV. Proc ION GPS-98, Nashville, TN, pp 31-38

Rothacher M (1994) Advanced techniques of GPS data processing at the AIUB in Berne. In: Crosilla F, Mussio L (eds) Metodi e Procedure di Modellizzazione e Trattamento dei Dati GPS. International Centre for Mechanical Sciences, Udine, pp 137153

Rothacher M (2001) Comparison of absolute and relative antenna phase center variations. GPS Solut 4(4):55-60

Rothacher M, Schaer S, Mervart L, Beutler G (1995) Determination of antenna phase center variations using GPS data. Gendt G. and Dick G. (eds) Proc 1995 IGS Workshop, GeoForschungs Zentrum Potsdam, Potsdam, pp 205-220

Saastamoinen J (1973) Contribution to the theory of atmospheric refraction. Bull Géod 107:13-34

Springer TA (2000) Common interests of the IGS and the IVS. Proc IVS 2000 General Meeting. Vandenberg, R. and Baver D. (eds) Kötzting, Germany, pp 296-305

Springer TA, Beutler G, Rothacher M (1999) Improving the orbit estimates of the GPS satellites. J Geod 73(3):147-157

Zhu SY, Massmann F-H, Yu Y, Reigber Ch (2003) Satellite antenna phase center offsets and scale errors in GPS solutions. J Geod 76(11-12): 668-672 